\title{
Multi-wavelength observations of microflares and emerging flux with YOHKOH/SXT
}

\author{
Toshifumi Shimizu ${ }^{1}$ \\ ${ }^{1}$ Solar-B project office, National Astronomical Observatory of Japan, National Institutes of \\ Natural Sciences, Mitaka, Tokyo 181-8588, JAPAN email: shimizu@solar.mtk.nao.ac.jp
}

\begin{abstract}
.
Soft X-ray observations by Yohkoh SXT have revealed that transient brightenings of small coronal loops frequently occur in active regions. Their estimated energy is $10^{24} \sim 10^{28}$ ergs and non-thermal behaviors may be observed in the large member of the brightenings. They are considered as soft X-ray signatures of microflares observed in hard X-rays. Lower coronal temperature plasma (1-2MK) produced by Yohkoh transient brightenings is simultaneously observed in EUV wavelengths by TRACE and SoHO EIT/CDS. Moreover, EUV observations have shown a lot of tinier transient brightenings without accompanying SXT transient brightenings. Coordinated observations with photospheric observations show that the location of Yohkoh transient brightenings is well localized in active regions, i.e., in emerging flux regions, around well-developed sunspots, and in the coronal bundles connecting the leading plage to the following plage regions. In some Yohkoh events, small-scale emergence of magnetic flux is well associated with their occurrence, suggesting that emerging flux plays a key role in triggering transient energy release in the corona. This paper reviews multi-wavelength observations coordinated with Yohkoh SXT observations for investigating the nature of SXT transient brightenings (SXR microflares) and their associated emerging flux. Finally, Solar-B space observatory is briefly introduced as a next powerful tool for multi-wavelength investigations.
\end{abstract}

\section{Introduction}

Soft X-ray Telescope (SXT) onboard the Yohkoh satellite made continuous observations in soft X-rays over 10 years (August 1991 - December 2001). Owing to its highly sensitive imaging with low scattering and high cadence, SXT has detected variety of small energy release events in the high-temperature $(\Varangle 3 \mathrm{MK})$ corona. In active regions, compact coronal loops show transient brightenings at various locations, and they are called transient loop brightenings or soft X-ray (SXR) microflares. During the observations, multi-wavelength observations were coordinated with ground-based as well as space-based observatories to understand small energy release events observed with SXT.

The purpose of this paper is to review multi-wavelength observations of transient brightenings (SXR microflares) with emphasis on coordinated SXT observations. Primary interests by the author (and also most of researchers) on SXT transient brightenings are: (1) How much can transient brightenings contribute to heating of the corona ( $(2)$, and (2) What is the origin of such small energy releases in solar atmosphere? For the second interest, it is essential to understand what SXT transient brightenings are related to similar events observed in other wavelengths, which are briefly reviewed in $\S 3$. Coordinated observations with high resolution magnetograms have revealed that smallscale emergence of magnetic fields from below the photosphere is well associated with the occurrence of transient loop brightenings. The role of emerging flux in the transient brightenings is discussed in $\S 4$. Finally, the $\operatorname{Solar}-B$ satellite is briefly mentioned in $\S$ 5 , which are highly expected as a next power tool for multi-wavelength investigations. 


\section{SXR microflares and coronal heating}

Microflare heating hypothesis is that the corona with $>1 \mathrm{MK}$ plasma is maintained by numerous number of small energy releases (magnetic reconnection events) in the corona, which are generated by braiding the magnetic fields due to photospheric convection motions (e.g., Parker 1988). The number distribution of SXT transient brightenings as a function of estimated energy was obtained by Shimizu (1995); The distribution can be represented by a power law function with index of -1.5 in $10^{27} \sim 10^{29}$ ergs, indicating that the distribution is just a lower extension from the flare distribution. Transient brightenings visible in SXT images provide at most a factor of 5 smaller than the energy required for the heating of the active-region corona and do not provide sufficient energy to the heating, although SXT transient brightenings are the main source for generating high-temperature $(>5 \mathrm{MK})$ plasma in the corona (Watanabe et al. 1995; Yoshida \& Tsuneta 1996).

More attentions have been paid in these days to nano- $\left(10^{24} \mathrm{erg}\right)$ and pico- $\left(10^{21} \mathrm{erg}\right)$ flare regions. With SoHO EIT and TRACE observations of EUV spectral $(1-2 \mathrm{MK})$ lines, the number distribution of EUV brightenings is determined in $10^{24} \sim 10^{26}$ ergs, giving that the distribution is represented by a power law with -1.8 (Aschwanden et al. 2000) or slightly steeper index (-2.4, Krucker \& Benz 1998; Parnell \& Jupp 1999). In the SXR bright bundles connecting the leading magnetic polarity region to the following polarity region, tiny variability can be commonly detected in time series of SXT images (Shimizu \& Tsuneta 1997; Katsukawa \& Tsuneta 2001). The variability is significant and much larger than the variation by X-ray photon statistics, suggesting that the variability would be a signature for giving some hits to the heating mechanism.

\section{Multi-wavelength observations of SXR microflares}

Coordinated observations in multi-wavelengths would provide key observational facts in helping us to understand the origin of SXR microflares (SXT transient brightenings). The following sub-sections describe typical properties of SXT transient brightenings derived from coordinated observations.

\subsection{Multi-temperature structures in hot plasma}

Coronal plasma produced by SXT transient brightenings has multi-temperature structures, which has been revealed by coordinated observations including SXT, SoHO EIT, and TRACE. SXT is sensitive to the plasma higher than $3 \mathrm{MK}$, whereas the other telescopes observes EUV spectral lines forming in 1-2 MK.

SXT transient brightenings have been compared with brightenings observed in EUV images (e.g., Berghmans, McKenzie \& Clette 2001). EUV brightenings are always observed when SXT transient brightenings occur. Weak EUV brightenings often do not have an SXR counterpart. Much more EUV events are observed than SXR events (373 EUV brightenings versus 41 SXR brightenings, Berghmans, McKenzie \& Clette 2001).

SXT transient brightenings are sometimes accompanied by a jet at their footpoint (e.g., Shimojo, Shibata, \& Harvey 1998). Jets are ejection of hot plasma from near brightening site with apparent velocity of $10-1000 \mathrm{~km} / \mathrm{s}$ (average $200 \mathrm{~km} / \mathrm{s}$ ). The X-ray intensity distribution along an X-ray jet often shows an exponential decrease with distance from the footpoint.

\subsection{Signature of accelerated non-thermal electrons}

Radio (micro-wave) as well as hard X-ray observations has revealed the existence of nonthermal electrons for large members of SXT transient brightenings (Gopalyswamy et al. 

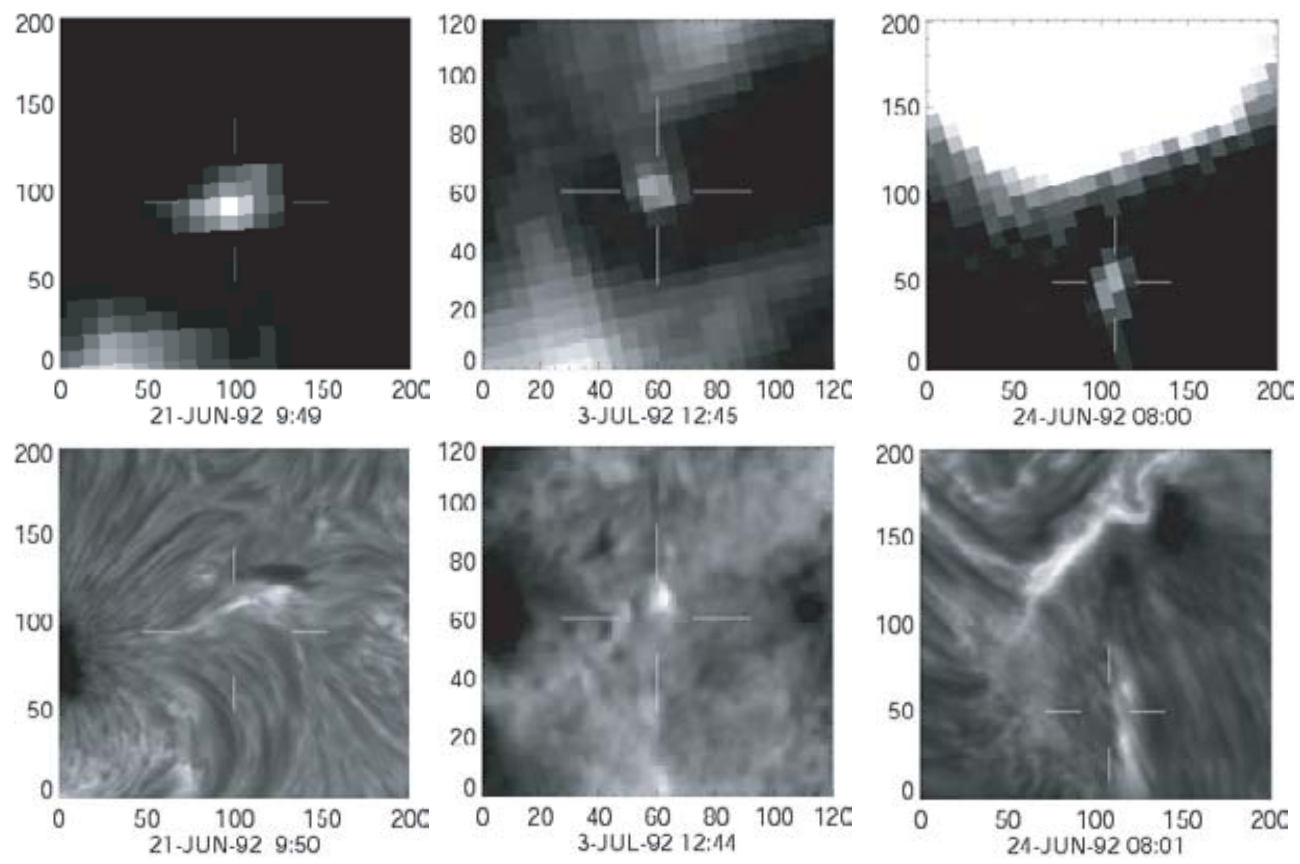

Figure 1. SXT transient brightenings (upper panels) and accompanying chromospheric brightening seen in simultaneous $\mathrm{H} \alpha$ center images (lower panels)

1994, White et al. 1995, Nitta 1997, Gary, Hartl, \& Shimizu 1997, Nindos, Kundu, \& White 1999). These observations suggest that SXT transient brightenings are just a small class of normal flares, although the non-thermal signatures have been detected only for intense brightenings. Owing to his higher sensitivity, RHESSI has newly provided hard X-ray spectral observations of such small events, although RHESSI was launched after the termination of Yohkoh observations. RHESSI observations has provided hard X-ray spectra (1-20keV) for small events, which can be fitted by the combination of a thermal spectrum and a power-law non-thermal spectra in early phase (Krucker et al. 2002; Liu et al. 2004). Interesting is that the index of the power law may be steeper $(-5 \sim-8)$ than that of normal flares. Note that the steep index is derived from the spectra in less than $20 \mathrm{keV}$, although the index of normal flares is usually derived with spectral fitting in the energy higher than $20 \mathrm{keV}$. This may be the first result suggesting a possible nature of microflares different from normal flares, which need to be understood.

\subsection{Chromospheric response to $S X R$ microflares}

Simultaneous $\mathrm{H} \alpha$ observations has revealed chromospheric response to SXT transient brightenings (Shimizu et al. 2002). Tiny brightenings are always observed in $\mathrm{H} \alpha$ center images, when coronal loops are brightened (Figure 1). Moreover, motions of chromospheric material, such as ejections, are sometimes observed. Appearance of tiny $\mathrm{H} \alpha$ kernels at footpoints of brightened coronal loops is a manifestation that the chromospheric material is heated due to non-thermal electrons impinging on the chromosphere and/or some heats are thermally conducted from the corona to the chromosphere. Tiny $\mathrm{H} \alpha$ brightenings are a good indicator for identifying more accurate position of footpoints 
of brightened coronal loops, which width would be much larger in the corona compared to the cross section at the lower atmosphere.

\subsection{Preferable locations in active regions}

SXT transient brightenings have been observed in active regions and X-ray bright points (XBP). Microflares have locations in active regions preferable for their occurrences. There are three areas where brightenings are well observed in active regions; Around the outer boundary of the penumbra of well-developed sunspots, in coronal bundles connecting between the leading and following magnetic polarity regions, and in and around emerging flux regions (EFR) where successive emergences of magnetic flux are significantly observed. Occurrence of many SXT transient brightenings around well-developed sunspots is associated with the development of satellite spots or pores (Leka et al. 1994). It does not appear that the brightenings are in close association with moving magnetic features (MMF). For further understanding, it is crucial to understand how magnetic fields involved in satellite spots develop with occurrence of microflares.

\subsection{Magnetic activities in high resolution magnetograms}

High resolution magnetograms simultaneously obtained at La Palma with SXT observations have revealed that small scale emergence of magnetic flux takes responsible for a type of SXR microflares (Shimizu et al. 2002). Point-like transient brightenings with X-ray source size less than 10 arcsec have been examined in detail, since the location of the magnetic fields involved in microflares can be well pinned down within 10 arcsec diameter at the photosphere below the X-ray source. The study revealed new appearance of magnetic poles in the magnetogram sequence for the half of the examined events. No apparent magnetic activity is not observed in the rest. The events without apparent magnetic activities are observed on rather intense magnetic regions where a pore and strong magnetic flux exist, suggesting that small magnetic activities may be obscured by relatively intense magnetic fields. The new magnetic flux observed with SXR brightenings is rather small with magnetic flux of $4 \times 10^{17} \sim 8 \times 10^{18} \mathrm{Mx}$.

In an example clearly showing the associated emerging activity (Figure 2, Shimizu et al, 2002), a small-scale emergence with the flux of $8 \sim 16 \times 10^{17} \mathrm{Mx}$ appears on the longitudinal magnetogram $\sim 10$ min prior to the onset of the SXR brightening, followed by the separation of a magnetic bipole with the speed of $2 \sim 2.8 \mathrm{~km} / \mathrm{s}$. A temporal relation between the first appearance of magnetic flux and the onset of SXT transient brightenings can be found in the events with emerging flux. In 6 events, a small-scale appearance of magnetic flux is observed $5 \sim 30 \mathrm{~min}$ before the onset of SXT events. A similar temporal relation can be found also in the event studied by Tang et al. (2001). This finding supports the vital role of emerging flux activity in triggering SXT transient brightenings.

\section{Role of emerging flux}

The La Palma - SXT coordinated observation indicates that emergence of magnetic flux probably plays a vital role in triggering SXT transient brightenings. An emerging flux model (e.g., Heywaerts, Priest, \& Rust 1977, Yokoyama \& Shibata 1996) is considered as an important process for converting magnetic energy into thermal and kinetic energy in the corona. In this model, a new emerging loop collides with pre-existing magnetic fields. A current sheet created between them develops an energy conversion process including magnetic reconnection. The detailed spatial relationship among newly emerging flux, soft X-ray source, and tiny $\mathrm{H} \alpha$ brightenings suggests that the merging flux model is 

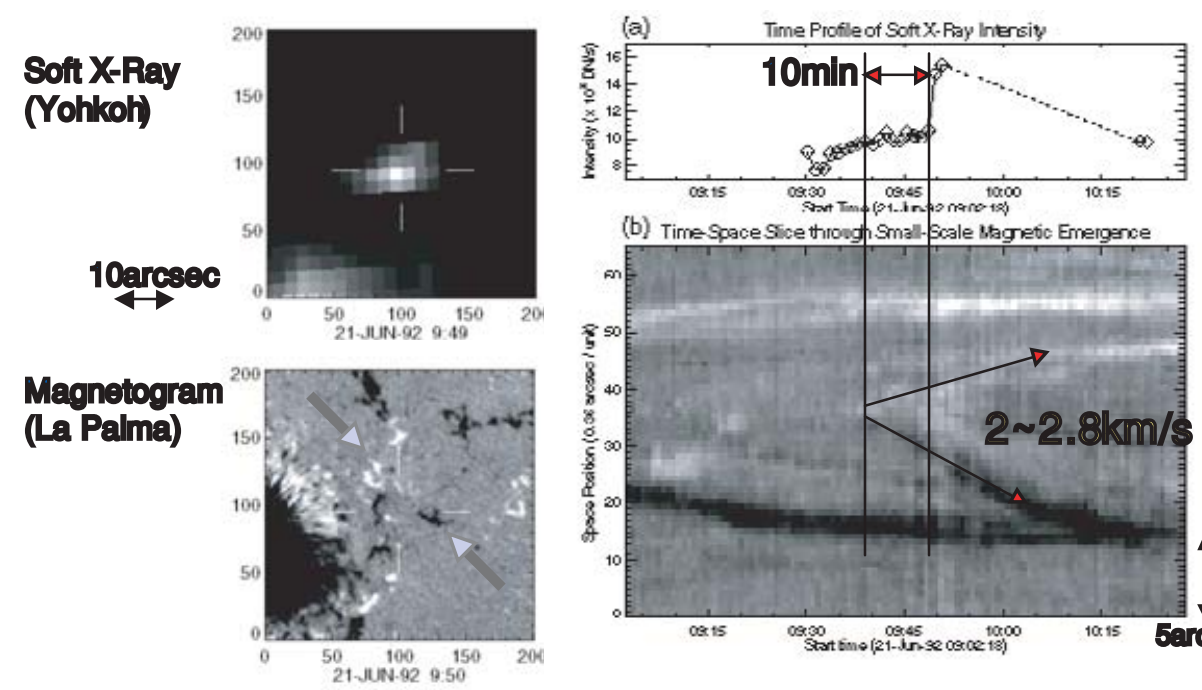

(b) Time-Space Sice through Small. Scale Stagrebic Emergance

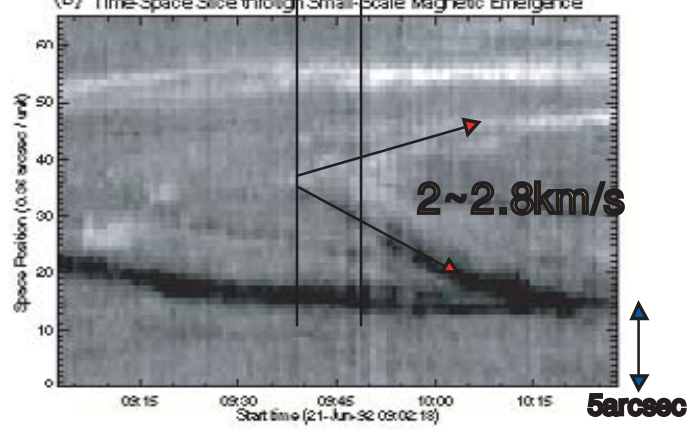

Figure 2. SXT transient brightening at 9:48UT and small-scale emergence of magnetic flux at 9:38UT on 21 June 2001. Details can be found in Shimizu et al. (2002)

preferable as the configuration of magnetic fields involved in the energy release (Shimizu 2002). For further investigating the physical process, we should observationally obtain further information on the two kinds of magnetic fields; pre-existing magnetic field and newly emerging magnetic field. The pre-existing magnetic field may be important for determining the environmental condition. The nature of emerging magnetic flux would be more important for triggering the energy release in the corona. Observational facts on the magnetic nature of emerging flux are lacking for discussing further. It is important to understand three-dimensional magnetic fields as well as their velocity fields involved in magnetic flux which has just appeared on the photosphere. A recent observational study has revealed that the newly emerging field just appeared on the photosphere is weak (300-500 gauss), horizontally oriented, upward $(<1 \mathrm{~km} / \mathrm{s}$ at the photosphere) field with large $(>80 \%)$ filling factor (Kubo, Shimizu, \& Lites 2003).

It should be noted that there are some observations showing magnetic cancellation for the mechanism on small energy releases in the corona (Chae et al. 1999; Zhang, Wang, \& Liu 2000, Yurchyshyn and Wang 2001, Sainz Dalda et al. 2004). The magnetic cancellation is apparently observed as decreasing magnetic poles on the longitudinal magnetogram signal. In this process, magnetic concentrations, which are initially unconnected with opposite polarity, converge and mutually lose flux. The magnetic field motions due to emergence may cause one of the emerging bipole to be cancelled with the nearby preexisting opposite flux concentration, which is same as the magnetic configuration of the emerging magnetic flux model. Magnetic reconnection may be triggered not just by the emerging of new magnetic flux, but also by convective motions of the photospheric gas. In this process, the magnetic flux moving due to convection collides with the opposite magnetic concentration. Moreover, magnetic canceling events could be just the submergence of magnetic flux, which do not produce the energy release in the corona. There may be a tendency that the canceling magnetic features are more reported from the observations with SoHO EIT and TRACE. This may mean that the events with magnetic emergence 
can more easily create the heat in higher temperature corona, which is well observed by SXT. On contrast, the events with canceling features may have magnetic reconnection at the low height, generating much plasma detectable with EIT and TRACE. It is noted that even when an emergence is well observed to be cancelled with pre-existing flux in magnetograms, no X-ray brightening is observed and instead a large surge is observed in $\mathrm{H} \alpha$ (Yoshimura et al. 2003). These observations suggest that the manifestation of energy release events strongly depend on the height of energy release where magnetic reconnection takes place.

\section{Solar-B as a next powerful tool}

Great advances will be expected with new observations in the near future, such as multi-wavelength observations performed by Solar-B satellite (Figure 3), which is scheduled for the launch in summer of 2006. Solar-B will carry three telescopes, providing coordinated multi-wavelength observations of the solar atomosphere including photosphere, chromosphere and the corona. Solar Optical Telescope (SOT) is the largest optical telescope (the clear aperture of primary mirror is $50 \mathrm{~cm}$ in diameter) ever to observe the Sun from the space and provides diffraction-limited $(0.2-0.3$ arcsec) imaging in $388-$ $668 \mathrm{~nm}$. The telescope has two focal-plane instruments (tunable filtergraph and spectropolarimeter) with capability of accurately measuring vector magnetic and velocity fields at the solar surface. X-Ray Telescope (XRT) is a grazing-incident telescope, providing the highest angular resolution imaging at $>3 \mathrm{MK}$ corona. Careful selection of X-ray analysis filters provides wide temperature coverage from below $1 \mathrm{MK}$ to above $10 \mathrm{MK}$, enabling us to understand the entire distribution of coronal plasma (Golub 2004). EUV Imaging Spectrometer (EIS) is a spectrograph with imaging capability in the 17-21 nm and 25$29 \mathrm{~nm}$ ranges, diagnosing the plasma conditions of dynamical coronal plasma precisely. These telescopes have been developed under the international collaborations among U.S., U.K. and Japan. Owing to sun-synchronous orbit, the telescopes can perform continuous observation without interruption for 8 months every a year. ESA has recently jointed the program to support data downlinks with polar station, giving significant increase in data volume.

\section{Acknowledgements}

The author would like to express his thanks to the organizers of the symposium for giving him this review opportunity. The work is supported by the Grant-in-Aid for Scientific Research No. 14740138.

\section{References}

Aschwanden, M.J., Tarbell, T.D, Nightingale, R.W., Schrijver, C.J., Title, A, Kankelborg, C.C., Martens, P., \& Warren, H.P. 2000, Astrophys. J. 535, 1047-1065.

Berghmans, D., McKenzie, D., \& Clette, F. 1998 A $\& A$ 369, 291-304.

Chae, J., Qiu, J., Wang, H., \& Goode, P.R. 1999 Astrophys. J. 513, L75-L78.

Gary, D.E., Hartl, M.D, \& Shimizu, T. 1997 Astrophys. J. 477, 958-968.

Golub, L. 2004 in these proceedings.

Gopalswamy, N., Payne, T.E.W., Schmahl, E.J., Kundu, M.R., Lemen, J.R., Strong, K.T., Canfield, R.C., \& de La Beaujardiere, J. 1994 Astrophys. J. 437, 522-528.

Heyvaerts, J., Priest, E.R., \& Rust, D.M. 1977 Astrophys. J. 216, 123-137.

Katsukawa, Y. \& Tsuneta, S. 2001, Astrophys. J. 557, 343-350.

Krucker, S., Christe, S., Lin, R.P., Hurford, G.J., \& Schwartz, R.A. 2002 Solar Physics 210, $445-456$. 


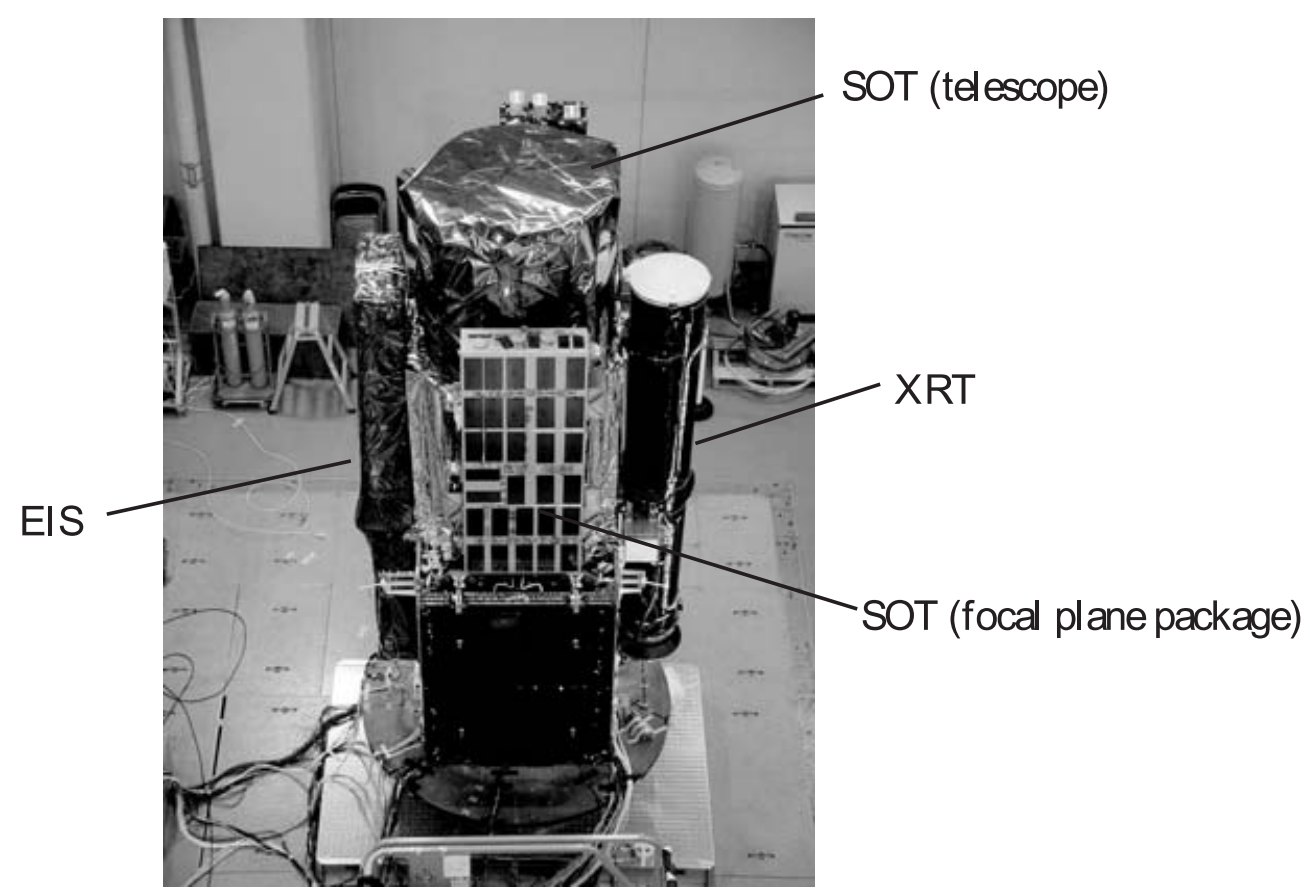

Figure 3. Solar-B satellite mechanical test model under environmental test (2002 May at JAXA/ISAS)

Krucker, S., Benz, A.O. 1998 Astrophys. J. 501, L213-L216.

Kubo, M., Shimizu, T., \& Lites, B.W. 2003 Astrophys. J. 595, 465-482.

Leka, K.D., van Driel-Gesztelyi, L., Nitta, N., Canfield, R.C., Mickey, D.L., Sakurai, T., \& Ichimoto, K. 1994 Solar Physics 155, 301-337.

Liu, C., Qiu, J., Gary, D.E., Krucker, S., \& Wang, H. 2004 Astrophys. J. 604, 442-448.

Nindos, A., Kundu, M.R., \& White, S.M. 1999, Astrophys. J. 513, 983-989.

Nitta, N. 1997 Astrophys. J. 491, 402-408.

Parker, E.N. 1988 Astrophys. J. 330, 474-479.

Parnell, C.E. and Jupp, P.E. 2000 Astrophys. J. 529, 554-569.

Sainz Dalda, A., Martinez Pillet, V. \& van Driel-Gesztelyi, L. 2004 in these proceedings.

Shimizu, T. 1995 PASJ 47, 251-263.

Shimizu, T. \& Tsuneta, S. 1997 Astrophys. J. 486, 1045-1057.

Shimizu, T. 2002, In Multi-Wavelength Observations of Coronal Structure and Dynamics (ed. P.C.H. Martens \& D.P. Cauffman). COSPAR Colloquia series, vol. 13, pp. 29-38. Pergamon.

Shimizu, T. Shine, R.A., Title, A.M., Tarbell, T.D., \& Frank, Z. 2002 Astrophys. J. 574, 10471088.

Shimojo, M., Shibata, K., \& Harvey, K.L. 1998 Solar Physics 178, 379-392.

Tang, Y.H., Li, Y.N., Fang, C., Aulanier, G., Schmieder, B., Demoulin, P., \& Sakurai, T. 2000 Astrophys. J. 534, 482-489.

Watanabe, T., Hara, H., Shimizu, T., Hiei, E., Bentley, R.D., Lang, J., Phillips, K.J.H., Pike, C.D., Fludra, A., \& Bromage, B.J.I. 1995 Solar Physics 157, 169-184.

White, S.M., Kundu, M.R., Shimizu, T., Shibasaki, K., \& Enome, S. 1995, Astrophys. J. 440, 435-440.

Yokoyama, T. \& Shibata, K. 1996 PASJ 48, 353-376. 
Yoshida, T. \& Tsuneta, S. 1996 Astrophys. J. 459, 342-346.

Yoshimura, K., Kurokawa, H., Shimojo, M., Shine, R. 2003 PASJ 55, 313-320.

Yurchyshyn, V.B. \& Wang, H. 2001 Solar Physics 202, 309-318.

Zhang, J., Wang, J., \& Liu, Y. 2000 A\&SA 361, 759-765. 